\title{
Growth Order of Eigenfunctions of Schrödinger Operators with Potentials Admitting Some Integral Conditions II - Applications -
}

By

\author{
Masaharu ARAI* and Jun UCHIYAmA**
}

\begin{abstract}
In this paper we give applications of M. Arai and J. Uchiyama [2, Theorems 1.1 and 1 . 2], which treat the sharp estimates of the growth orders of the eigenfunctions of the Schrödinger operators with potentials oscillating violently at infinity. We can generalize the results of G.B. Khosrovshahi, H.A. Levine and L.E. Payne (Trans. Amer. Math. Soc., 253 (1979), 211228) and H. Kalf and V.K. Kumar (Trans. Amer. Math. Soc., 275 (1983), 215-229). Also we generalize the result of S. Agmon (J. Analyse Math., 23 (1970), 1-25).
\end{abstract}

\section{§o. Introduction}

Let us consider a not identically vanishing solution $u(x) \in H_{\text {loc }}^{2}(\Omega)$ of a second order elliptic equation

$$
-\sum_{j=1}^{n}\left(\partial_{j}+\sqrt{-1} b_{j}(x)\right)^{2} u(x)+\left(q_{1}(x)+q_{2}(x)\right) u(x)=0
$$

for $x \in \Omega:=\left\{x\left|x \in \boldsymbol{R}^{n},\right| x \mid>R_{0}\right\}$, where $\partial_{j}=\partial /\left(\partial x_{j}\right), b_{j}(x)$ and $q_{1}(x)$ are realvalued functions, $q_{2}(x)$ is a complex-valued function.

In this paper we give applications of Arai-Uchiyama [2, Theorems 1.1 and 1.2]. So we rewrite their assumptions and results for completeness of this paper. We use the same notation as in Arai-Uchiyama [2].

Assumptions. Let us consider the equation (0.1). We assume that there exist some real-valued functions $\psi_{i}(r) \in C^{0}\left[R_{0}, \infty\right), \sigma_{i}(r), \eta_{i}(r) \in C^{1}\left[R_{0}, \infty\right)(i$ $=1,2), \tau(r) \in C^{0}\left[R_{0}, \infty\right)$ and some constants $a_{i}(i=1,2)$ satisfying the follow-

Communicated by T. Kawai, January 11, 1995.

1991 Mathematical Subject Classification : Primary 35P15; Secondary 35J10.

*Department of Mathematics and Physics, Faculty of Science and Engineering, Ritsumeikan University, Kyoto 603, Japan.

${ }^{* *}$ Kyoto Institute of Technology, Matsugasaki, Sakyo-ku, Kyoto 606, Japan 
ing conditions (B.1)-(F.2) :

(B.1) each $b_{j}(x)$ is a real-valued function ;

(B.2) for any $w(x) \in H_{l o c}^{1}(\Omega)$ we have $b_{j}(x) w(x),\left(\partial_{j} b_{k}(x)\right) w(x) \in L_{l o c}^{2}(\Omega)$;

(C.1) $q_{1}(x)$ is a real-valued function;

(C.2) for any $w(x) \in H_{l o c}^{1}(\Omega)$ we have $q_{1}(x)|w(x)|^{2} \in L_{l o c}^{1}(\Omega)$;

(C.3) for any $w(x) \in H_{l o c}^{1}(\Omega)$ we have $\partial_{r} q_{1}(x) \cdot|w(x)|^{2} \in L_{l o c}^{1}(\Omega)$;

(D.1) $q_{2}(x)$ may be a complex-valued function;

(D.2) for any $w(x) \in H_{l o c}^{1}(\Omega)$ we have $q_{2}(x)|w(x)|^{2} \in L_{l o c}^{1}(\Omega)$;

(E.1) $\sigma_{i}(r)$ is bounded in $\left[R_{0}, \infty\right)$ and $\sigma_{i}(r)>0(i=1,2)$ for any $r \geq R_{0}$;

(E.2) $\eta_{i}(r)$ is bounded in $\left[R_{0}, \infty\right)$ and we have for any $r \geq R_{0}$

$$
\eta_{i}(r)<2(i=1,2) \text {; }
$$

(E.3) $\quad \psi_{i}(r)>0(i=1,2)$ for any $r \geq R_{0}$;

(E.4) $\lim _{r \rightarrow \infty} r^{-1} \psi_{i}(r) \sigma_{i}(r)^{-1}=0(i=1,2)$;

(E.5) $\quad r\left\{\sigma_{1}^{\prime}(r)-\sigma_{2}^{\prime}(r)-\left(\eta_{1}^{\prime}(r)-\eta_{2}^{\prime}(r)\right)\right\}$ is bounded in $\left[R_{0}, \infty\right)$;

(E.6) there exists some constant $C_{1} \geq 1$ such that for any $r \geq R_{0}$

$$
C_{1}^{-1} \sigma_{2}(r) \leq \tau(r) \leq C_{1}
$$

(E.7) $\lim _{r \rightarrow \infty} \psi_{2}(r)^{2} \sigma_{2}(r)^{-1} \exp \left(\int_{R_{0}}^{r} \frac{r(t)-\eta_{2}(t)}{t} d t\right)=0$;

(F.1) $\quad a_{1}>1, a_{2}>0$;

(F.2) $\limsup _{r \rightarrow \infty} \phi_{i}(r)^{2} \sigma_{i}(r)^{-1}\left[r \partial_{r} q_{1}+\eta_{i}(r) q_{1}+a_{i} \sigma_{i}(r)^{-1}\left|r q_{2}-Q_{i}^{\prime}(r)\right|^{2}\right.$

$$
\left.+\left(2-\eta_{i}(r)\right)^{-1}|B x|^{2}\right]<0(i=1,2),
$$

where we put

$$
\begin{aligned}
& Q_{i}(r)=4^{-1}\left(\eta_{i}(r)-\sigma_{i}(r)\right), \\
& B(x)=\left(\partial_{j} b_{k}(x)-\partial_{k} b_{j}(x)\right) \text { is an } n \times n \text { matrix. }
\end{aligned}
$$

Theorems 1.1 and 1.2 of Arai-Uchiyama [2] are as follows:

Theorem 0.1. Let $u(x) \in H_{\text {loc }}^{2}(\Omega)$ satisfy the equation (0.1). Assume that (B)-(F) hold. If $\operatorname{supp}[u]$ is not a compact set in $\bar{\Omega}$, then we have 


$$
\liminf _{R \rightarrow \infty} \Phi_{1}(R) \int_{|x|=R}\left[\left|D_{r} u\right|^{2}+\left\{r^{-2}+\left(q_{1}\right)_{-}\right\}|u|^{2}\right] d S>0
$$

where

$$
\begin{aligned}
& (f)_{-}(x)=\max \{0,-f(x)\} \geq 0 \text { for a real-valued function } f(x), \\
& \Phi_{1}(r)=\exp \left(\int_{R_{0}}^{r} \frac{\sigma_{1}(t)+\eta_{1}(t)}{2 t} d t\right) .
\end{aligned}
$$

Theorem 0.2. In addition to the assumptions of Theorem 0.1, assume that

(G) there exist some real-valued bounded function $Q_{0}(x) \in C^{1}(\bar{\Omega})$, some positive and non-decreasing function $p(r) \in C^{1}\left[R_{0}, \infty\right)$ and some constant $0<$ $d_{1}<1$ satisfying the following (G.1)-(G.3):

(G.1) for any $w(x) \in C_{0}^{\infty}(\Omega)$ we have

$$
\begin{aligned}
& \int_{\Omega}\left\{\left(q_{1}\right)_{-}(x)+\left(\operatorname{Re}\left[q_{2}\right]-r^{-1} \partial_{r} Q_{0}\right)_{-}(x)\right\}|w(x)|^{2} d x \\
& \quad \leq \int_{\Omega}\left\{d_{1}|\nabla w(x)|^{2}+p(r)|w(x)|^{2}\right\} d x
\end{aligned}
$$

(G.2) $\liminf _{r \rightarrow \infty} p(r) p(r+1)^{-1}>0$;

(G.3) $p^{\prime}(r)^{2} p(r)^{-3}$ is bounded in $\left[R_{0}, \infty\right)$.

Then we have
(1) $\liminf _{R \rightarrow \infty} p(R) \Phi_{1}(R) \int_{R<|x|<R+1}|u(x)|^{2} d x>0$;
(2) moreover if $\int_{R_{0}}^{\infty} p(R)^{-1} \Phi_{1}(R)^{-1} d R=\infty$ then $u(x) \notin L^{2}(\Omega)$.

The aims of this paper are the following:

(1) to give a generalization of the results of Khosrovshahi-Levine-Payne [7] and Kalf-Kummar [5] (Theorem 1.1);

(2) to give a generalization of the result of Agmon [1] (Theorem 1.4) ;

(3) to check that our results cover all the ones given by Uchiyama-Yamada [9] (Application 1.6).

We will apply our Theorem 0.2 to von Neumann-Wigner [8] example in other article. 


\section{§1. Applications of Theorems 0.1 and 0.2}

Theorem 1.1. Let $b_{j}(x) \in C^{1}(\bar{\Omega})(j=1, \cdots, n), V_{1}(x) \in C^{1}(\bar{\Omega})$ and $V_{3}(r)$ $\in C^{0}\left[R_{0}, \infty\right)$ be real-valued functions, and $V_{2}(x) \in C^{0}(\bar{\Omega})$ be a complex-valued function. Let $u(x) \in H_{\text {loc }}^{2}(\Omega) \cap L^{2}(\Omega)$ satisfy

$$
-\sum_{j=1}^{n}\left(\partial_{j}+\sqrt{-1} b_{j}(x)\right)^{2} u(x)+\left\{V_{1}(x)+V_{2}(x)+V_{3}(r)\right\} u(x)=\lambda u(x)
$$

for $x \in \Omega:=\left\{x|| x \mid>R_{0}\right\}$. Let

$$
Q(r)=\int_{R_{0}}^{r} t V_{3}(t) d t
$$

We assume:

(1) $V_{1}(x)$ is bounded on $\bar{\Omega}$,

(2) $\limsup _{r \rightarrow \infty} V_{1}(x)=0$,

(3) $0 \leq \limsup _{r \rightarrow \infty} r \partial_{r} V_{1}(x)=L<\infty$,

(4) $\limsup _{r \rightarrow \infty}\left\{\left|r V_{2}(x)\right|^{2}+|B(x) x|^{2}\right\}=K^{2}<\infty$,

(5) $\limsup _{r \rightarrow \infty} Q(r)-\liminf _{r \rightarrow \infty} Q(r)=M<1$,

(6) $\lambda>\Lambda:=\frac{1}{2} \cdot \frac{1}{1-M^{2}}\left[K^{2}+L+\sqrt{K^{2}\left(K^{2}+2 L\right)+L^{2} M^{2}}\right]$.

Then we have $u(x) \equiv 0$ on $\Omega$. (The condition $L \geq 0$ in (3) is automatically satisfied by the condition (1).)

Remark 1.2. Noting the results given in Arai-Uchiyama $[2, \S 8]$, we can treat the case $V_{3}=V_{3}(x)$. In this case we can replace (4) and (5) with the following $(4)^{\prime}$ and $(5)^{\prime}$ respectively.

$(4)^{\prime} \quad \limsup _{r \rightarrow \infty}\left\{\left|r V_{2}(x)\right|^{2}+|\nabla Q(x)|^{2}-\left|\partial_{r} Q(x)\right|^{2}+|B(x) x|^{2}\right\}=K^{2}<\infty$, where

$$
Q(x)=\int_{R_{0}}^{|x|} t V_{3}(t \bar{x}) d t(\widehat{x}=x /|x|)
$$

(5) $\quad \limsup _{r \rightarrow \infty} Q(x)-\liminf _{r \rightarrow \infty} Q(x)=M<1$.

Remark 1.3. We state several results obtained before from which we have 
the conclusion that $\lambda(>\Lambda)$ is not an eigenvalue. ( $\Lambda$ defined above will be denoted as $\Lambda_{\mathrm{AU}}$.) Kato [6] considered the case $V_{1}(x) \equiv V_{3}(r) \equiv b_{j}(x) \equiv 0$ and gave

$$
\Lambda_{\mathrm{K}}=K^{2}
$$

Agmon [1] considered the case $V_{3}(r) \equiv b_{j}(x) \equiv 0, K=0$ and gave

$$
\Lambda_{\mathrm{A}}=\frac{L}{2} \text {. }
$$

Eastham-Kalf $\left[3\right.$, p. 187] considered the case $V_{3}(r) \equiv 0$ and gave

$$
\Lambda_{\mathrm{EK}}=\frac{1}{2}\left\{\widetilde{K}^{2}+L+\sqrt{\widetilde{K}^{2}\left(\widetilde{K}^{2}+2 L\right)}\right\}
$$

where $\widetilde{K}=\limsup _{r \rightarrow \infty}\left\{r\left|V_{2}(x)\right|+|B(x) x|\right\}(\geq K)$. Khosrovshahi-Levine-Payne [7] considered the case $b_{j}(x) \equiv 0$ and gave under the condition $M<4^{-1}$

$$
\Lambda_{\mathrm{KLP}}=\max \left\{\left[\frac{K+\sqrt{K^{2}+2 L(1-2 M)}}{2(1-2 M)}\right]^{2}, \frac{2 K^{2}+L(1-4 M)}{2(1-4 M)^{2}}\right\} .
$$

Kalf-Kumar [5] treated the case $V_{3}=V_{3}(x)$ and $\lim _{r \rightarrow \infty}\left\{|\nabla Q(x)|^{2}-\left|\partial_{r} Q(x)\right|^{2}\right\}=0$, and gave under the condition $M<2^{-1}$

$$
\Lambda_{\mathrm{KK}}=\left[\frac{\widetilde{K}+\sqrt{\widetilde{K}^{2}+2 L(1-2 M)}}{2(1-2 M)}\right]^{2} .
$$

(We remark that we correct the error in the representation of $\Lambda_{\mathrm{KLP}}$ given in Khosrovshahi-Levine-Payne [7] and Kalf-Kumar [5].) It is obvious that $\Lambda_{\mathrm{KK}}$ $=\Lambda_{\mathrm{EK}}$ under the assumption of Eastham-Kalf [3] and that $\Lambda_{\mathrm{EK}}, \Lambda_{\mathrm{KLP}}, \Lambda_{\mathrm{KK}}$ and $\Lambda_{\mathrm{AU}}$ reduce to $\Lambda_{\mathrm{K}}$ or $\Lambda_{\mathrm{A}}$ under the Kato's or Agmon's assumptions, respectively. After careful consideration, noting Remark 1.2, we can see $\Lambda_{\mathrm{KLP}} \geq \Lambda_{\mathrm{KK}} \geq \Lambda_{\mathrm{AU}}$.

Theorem 1.4 (Generalization of Agmon's Theorem.) Let $u(x) \in$ $H_{\text {loc }}^{2}(\Omega)$ satisfy

$$
\begin{aligned}
& -\sum_{j=1}^{n}\left(\frac{\partial}{\partial x_{j}}+\sqrt{-1} b_{j}(x)\right)^{2} u(x)+\left\{V_{1}(x)+V_{2}(x)+V_{3}(r)\right\} u(x) \\
& \quad=\lambda u(x) \text { in } \Omega
\end{aligned}
$$

and $u(x) \neq 0$ in $\Omega:=\left\{x|| x \mid>R_{0}\right\}$. Assume

(AG.1)

$\lambda$ is a real constant,

$\lambda-V_{1}(x) \in C^{0}(\bar{\Omega})$ is a positive function possessing a continuous radial 
derivative,

$V_{2}(x) \in C^{0}(\bar{\Omega})$ is a complex-valued function,

$V_{3}(r) \in C^{1}\left[R_{0}, \infty\right)$ is a real-valued function.

each $b_{j}(x) \in C^{1}(\bar{\Omega})$ is a real-valued function,

(AG.2) There exist a number $\alpha>0$, continuous non-negative functions $\zeta_{1}(r)$, $\zeta_{2}(r)$ and $\mu(r)$ defined on $r \geq R_{0}$, and a real-valued function $Q(r) \in$ $C^{1}\left[R_{0}, \infty\right)$, satisfying
(i) $\int_{R_{0}}^{\infty} \zeta_{i}(r) d r<\infty \quad(i=1,2)$,
(ii) $\limsup _{r \rightarrow \infty} r\left\{\zeta_{1}(r)+\zeta_{2}(r)\right\}<2 \alpha$,
(iii) $-\partial_{r} V_{1}(x)+\left(\frac{2(1-\alpha)}{r}+\zeta_{1}(r)\right)\left(\lambda-V_{1}(x)\right) \geq 0$ for $r \geq R_{0}$,
(iv) $\left|V_{2}(x)\right| \leq \zeta_{2}(r)\left(\lambda-V_{1}(x)\right)^{1 / 2}$ for $r \geq R_{0}$,
(v) $Q^{\prime}(r)=r V_{3}(r)$ for $r \geq R_{0}$,
(vi) $|Q(r)| \leq r \mu(r)\left\{\zeta_{1}(r)+\zeta_{2}(r)\right\}$ for $r \geq R_{0}$,
(vii) $|B(x) x|^{2} \leq r \mu(r)\left\{\zeta_{1}(r)+\zeta_{2}(r)\right\}\left(\lambda-V_{1}(x)\right)$ for $r \geq R_{0}$,
(viii) $\lim _{r \rightarrow \infty} \mu(r)=0$.

Then we have

$$
\liminf _{R \rightarrow \infty} R^{-\alpha} \int_{R_{0}<|x|<R}\left(\lambda-V_{1}(x)\right)|u(x)|^{2} d x>0 .
$$

Remark 1.5. Agmon[1] showed the above result for $b_{j}(x) \equiv 0$ and $V_{3}(r)$ $\equiv 0$ (, which means $Q(r) \equiv 0$ ), under the above conditions (i), (iii) and the following more restricted conditions than ours

$$
\begin{aligned}
& \text { (ii)' }^{\prime} \quad \limsup _{r \rightarrow \infty} r\left\{\zeta_{1}(r)+\zeta_{2}(r)\right\}<\alpha, \\
& \text { (iv)' } \max _{|x|=r}\left|V_{2}(x)\right| \leq \zeta_{2}(r) \min _{|x|=r}\left(\lambda-V_{1}(x)\right)^{1 / 2} \text { for } r \geq R_{0} .
\end{aligned}
$$

(v)-(viii) did not relate to his problem.

In the similar fashion as Remark 1.2, we can treat the case $V_{3}=V_{3}(x)$. In this case we can replace (v) and (vii) with the following (v)' and (vii)'.

$$
\begin{array}{ll}
\text { (v) } & \partial_{r} Q(x)=r V_{3}(x) \text { for } r \geq R_{0}, \\
\text { (vii)' } \quad|\nabla Q(x)|^{2}-\left|\partial_{r} Q(x)\right|^{2}+|B(x) x|^{2} \\
\quad \leq r \mu(r)\left\{\zeta_{1}(r)+\zeta_{2}(r)\right\}\left(\lambda-V_{1}(x)\right) \text { for } r \geq R_{0} .
\end{array}
$$


Application 1.6. Here we refer to the results of Uchiyama-Yamada [9]. Though they treated more general second-order elliptic equations and we can show that our theory in $[2, \S 8]$ recovers their results completely, we restrict ourselves to the case of the equation $(0,1)$ for simplicity. In this case their assumptions become as follows.

(UY.1) our Assumptions (B)-(D) are assumed.

(UY.2) there exist some real constants $0<\alpha<\beta, \tilde{\gamma}_{i} \in \boldsymbol{R}, \widetilde{a}_{1}>1, \tilde{a}_{2}>0$, $\widetilde{b}_{i}>1, \quad 0<\widetilde{d}_{1}<1, \quad \delta_{1} \in \boldsymbol{R}, \delta_{2} \leq \beta-2, \quad \widetilde{C}_{i}>0 \quad(i=1,2)$ and some real-valued functions $\sigma(r) \in C^{1}(\bar{\Omega}), \eta(r) \in C^{1}(\bar{\Omega})$ such that the following (UY.3)-(UY.13) hold ;

(UY.3) $\sigma(r)$ is bounded and $\sigma(r)>0$ for any $x \in \bar{\Omega}$;

(UY.4) $\eta(r)$ is bounded in $\bar{\Omega}$ and $\widetilde{\gamma}_{1}+\limsup _{r \rightarrow \infty} \eta(r)<2$;

(UY.5) $\quad(2-2 \beta<) 2-2 \alpha<\widetilde{\gamma}_{2}+\liminf _{r \rightarrow \infty} \eta(r) \leq \widetilde{\gamma}_{2}+\limsup _{r \rightarrow \infty} \eta(r)<2$;

(UY.6) $\lim _{r \rightarrow \infty} r^{\beta-\alpha} \sigma(r)=\infty$;

(UY.7) $\lim _{r \rightarrow \infty} r^{1-\beta} \sigma(r)^{-1} \sigma^{\prime}(r)=0$ and $\lim _{r \rightarrow \infty} r^{1-\beta} \sigma(r)^{-1} \eta^{\prime}(r)=0$;

(UY.8) $\limsup _{r \rightarrow \infty} r^{2-2 \beta} \sigma(r)^{-1}\left[r \partial_{r} q_{1}+\left(\tilde{\gamma}_{i}+\eta(r)\right) q_{1}\right.$

$$
\begin{aligned}
& \left.\quad+\widetilde{a}_{i} \sigma(r)^{-1}\left|r q_{2}\right|^{2}+\widetilde{b}_{i}\left(2-\widetilde{\gamma}_{i}-\eta(r)\right)^{-1}|B x|^{2}\right] \\
<0 & (i=1,2)
\end{aligned}
$$

(UY.9) for any $w(x) \in C_{0}^{\infty}(\Omega)$ we have

$$
\int_{\Omega}\left(q_{1}\right)_{-}|w|^{2} d x \leq \int_{\Omega}\left\{\widetilde{d}_{1}|\nabla w|^{2}+\widetilde{C}_{1} r^{\delta_{1}}|w|^{2}\right\} d x
$$

(UY.10) for any $x \in \bar{\Omega}$ we have $\left(\operatorname{Re}\left[q_{2}\right]\right)_{-}(x) \leq \widetilde{C}_{2} \min \left\{r^{\delta_{2}}, r^{\beta-2} \sigma(r)\right\}$.

Then they gave the following results for the solution $u(x) \in H_{l o c}^{2}(\Omega)$ of $(0.1)$ whose support is not a compact set in $\bar{\Omega}$ :

$$
\begin{aligned}
& \liminf _{r \rightarrow \infty} R^{\tilde{\gamma}_{1} / 2} \Phi(R) \int_{|x|=R}\left[\left|D_{r} u\right|^{2}+\left\{r^{-2}+\left(q_{1}\right)_{-}\right\}|u|^{2}\right] d S>0, \\
& \liminf _{R \rightarrow \infty} R^{\left(\tilde{\gamma}_{1} / 2\right)+\max \left\{0, \delta_{1}, \delta_{2}\right\}} \Phi(R) \int_{R<|x|<R+1}|u|^{2} d x>0,
\end{aligned}
$$

where

$$
\Phi(R)=\exp \left(\int_{R_{0}}^{R} \frac{\sigma(t)+\eta(t)}{2 t} d t\right)
$$

Apply our Theorems 0.1 and 0.2 to the above circumstances, and we can 
show their results. So our Assumptions treat wider classes of potentials than those of Uchiyama-Yamada [9]. In fact let for $i=1,2$

$$
\begin{aligned}
& \psi_{i}(r)=r^{1-\beta}, \\
& \sigma_{i}(r)=\sigma(r), \\
& \eta_{i}(r)=\tilde{\gamma}_{i}+\eta(r) .
\end{aligned}
$$

By (UY.3)-(UY.5), (E.1) and (E.2) hold. It is obvious that (E.3) and (E.5) hold. By (UY.6) and $\alpha>0$, we can see that (E.4) holds. Noting (UY.5), we choose $\tau_{0}$ $>0$ satisfying $2-2 \alpha+\tau_{0}<\widetilde{\gamma}_{2}+\liminf _{r \rightarrow \infty} \eta(r)$, and put $\tau(t) \equiv \tau_{0}$, by which and (UY.3) we have (E.6). Then for sufficiently large $r$ we have $2-2 \alpha+\tau_{0}-\left(\tilde{\gamma}_{2}\right.$ $+\eta(r)) \leq 0$. Noting (UY.3), (UY.6) and

$$
\begin{aligned}
& \psi_{2}^{2} \sigma_{2}^{-1} \exp \left(\int_{R_{0}}^{r} \frac{r(t)-\eta_{2}(t)}{t} d t\right) \\
& \quad=R_{0}^{2-2 \alpha} r^{2(\alpha-\beta)} \sigma(r)^{-2} \cdot \sigma(r) \cdot \exp \left(\int_{R_{0}}^{r} \frac{2-2 \alpha+\tau_{0}-\left(\tilde{\gamma}_{2}+\eta(t)\right)}{t} d t\right),
\end{aligned}
$$

we have (E.7). Let $a_{i}$ satisfy $\tilde{a}_{1}>a_{1}>1$ and $\tilde{a}_{2}>a_{2}>0$. Then we have (F.2) from (UY.8) and the fact $\psi_{i} \sigma_{i}^{-1} Q_{i}^{\prime}=0(1)$, which follows from (UY.7). Let $d_{1}=$ $\widetilde{d}_{1}, Q_{0}(x) \equiv 0$ and $p(r)=\left(\widetilde{C}_{1}+\widetilde{C}_{2}\right) r^{\max \left\{0, \delta_{1}, \delta_{2}\right\}}$. Then by (UY.9) and (UY.10) we have (G). Therefore, noting $\Phi_{1}(r)=\left(r / R_{0}\right)^{\tilde{\gamma}_{1} / 2} \Phi(r)$, by our Theorems 0.1 and 0 . 2 we have their results.

\section{Remark 1.7.}

(1) Uchiyama-Yamada [9, Example 1.7, Cases 2 and 3] gave the examples such that $\tilde{\gamma}_{1}+\lim _{r \rightarrow \infty} \eta(r)=2-2 \beta$. Noting (UY.5), we cannot, in general, put $\tilde{\gamma}_{1}$ $=\tilde{\gamma}_{2}$.

(2) The above condition (UY.7) can be replaced with

$$
(\mathrm{UY} .7)^{\prime} \lim _{r \rightarrow \infty} r^{1-\beta} \sigma(r)^{-1}(\eta(r)-\sigma(r))^{\prime}=0 .
$$

In fact in the above argument we use (UY.7) only to show $\psi_{i} \sigma_{i}^{-1} Q_{i}^{\prime}=0(1)$, which also comes out from (UY.7)'.

\section{§2. Proof of Theorem 1.1}

Lemma 2.1. Let $K \geq 0, L \geq 0,0 \leq M<1$ be constants and

$$
f(t)=\frac{1}{2}\left\{\frac{K^{2}+2 L}{t}+\frac{K^{2}}{2-t}\right\}(0<t<2) .
$$

Then we have 


$$
\inf _{0<\beta<2-2 M}(\sup \{f(t) \mid \beta \leq t \leq \beta+2 M\})=\Lambda
$$

where

$$
\Lambda=\frac{1}{2} \cdot \frac{1}{1-M^{2}}\left\{K^{2}+L+\sqrt{K^{2}\left(K^{2}+2 L\right)+L^{2} M^{2}}\right\},
$$

which is the one given in the statement of Theorem 1.1.

Proof. First let us consider the case $K>0$. Put

$$
t_{0}=\frac{2 \sqrt{K^{2}+2 L}}{K+\sqrt{K^{2}+2 L}} .
$$

Then we have

$$
\left\{\begin{array}{l}
1 \leq t_{0}<2, \\
\min _{0<t<2} f(t)=f\left(t_{0}\right)=2^{-1}\left\{K^{2}+L+K \sqrt{K^{2}+2 L}\right\} \\
f(t) \text { is continuous in }(0,2), \\
f(t) \text { is decreasing in }\left(0, t_{0}\right) \text { and increasing in }\left(t_{0}, 2\right) .
\end{array}\right.
$$

Let us consider the case $0<M<1$. Noting $\lim _{t \downarrow 0} f(t)=\lim _{t \uparrow 2} f(t)=\infty$, we apply the intermediate-value theorem. Then for any $s \in\left(f\left(t_{0}\right), \infty\right)$ there uniquely exist some $t_{1}=t_{1}(s) \in\left(0, t_{0}\right)$ and $t_{2}=t_{2}(s) \in\left(t_{0}, 2\right)$ such that $s=f\left(t_{1}(s)\right)=f\left(t_{2}(s)\right)$. We can see that $t_{2}(s)-t_{1}(s)$ is a positive continuous increasing function of $s \in$ $\left(f\left(t_{0}\right), \infty\right), \lim _{s \downarrow f\left(t_{0}\right)}\left(t_{2}(s)-t_{1}(s)\right)=0, \lim _{s \rightarrow \infty}\left(t_{2}(s)-t_{1}(s)\right)=2$. Noting $0<M<1$, we apply the intermediate-value theorem again. Then there uniquely exists some $s_{0}$ $\in\left(f\left(t_{0}\right), \infty\right)$ such that $t_{2}\left(s_{0}\right)-t_{1}\left(s_{0}\right)=2 M$. We put $\beta_{1}=t_{1}\left(s_{0}\right)$. Since $t_{1}\left(s_{0}\right)+2 M$ $=t_{2}\left(s_{0}\right)<2$ we have $\beta_{1} \in(0,2-2 M)$ and $f\left(\beta_{1}\right)=f\left(\beta_{1}+2 M\right)$.

If $M=0$, then we put $\beta_{1}=t_{0}$.

So for $M \geq 0$, we have $0<\beta_{1}<2-2 M$ and

$$
\begin{aligned}
& \min _{0<\beta<2-2 M}(\sup \{f(t) \mid \beta \leq t \leq \beta+2 M\}) \\
& \quad=\min _{0<\beta<2-2 M}(\max \{f(\beta), f(\beta+2 M)\}) \\
& =f\left(\beta_{1}\right) .
\end{aligned}
$$

By $f\left(\beta_{1}\right)=f\left(\beta_{1}+2 M\right)$ we have

$$
L \beta_{1}^{2}-2 \beta_{1}\left\{K^{2}+(2-M) L\right\}+2(1-M)\left(K^{2}+2 L\right)=0 .
$$

Thus

$$
\beta_{1}= \begin{cases}L^{-1}\left\{K^{2}+(2-M) L-\sqrt{K^{2}\left(K^{2}+2 L\right)+L^{2} M^{2}}\right\} & \text { if } L>0 \\ 1-M & \text { if } L=0\end{cases}
$$


Since for $L \geq 0$

$$
\begin{aligned}
& 2^{-1} \beta_{1}^{-1}\left(K^{2}+2 L\right) \\
& \quad=4^{-1}(1-M)^{-1}\left\{\sqrt{K^{2}\left(K^{2}+2 L\right)+L^{2} M^{2}}+K^{2}+(2-M) L\right\} \\
& 2^{-1}\left(2-\beta_{1}\right)^{-1} K^{2} \\
& \quad=4^{-1}(1+M)^{-1}\left\{\sqrt{K^{2}\left(K^{2}+2 L\right)+L^{2} M^{2}}+K^{2}-L M\right\}
\end{aligned}
$$

we have $f\left(\beta_{1}\right)=\Lambda$.

Next let us consider the case $K=0$. In this case we have $f(t)=L t^{-1}$ and

$$
\begin{array}{r}
\inf _{0<\beta<2-2 M}(\sup \{f(t) \mid \beta \leq t \leq \beta+2 M\}) \\
=\inf _{0<\beta<2-2 M} f(\beta)=\frac{1}{2} \cdot \frac{L}{1-M}=\Lambda .
\end{array}
$$

Now we give the proof of Theorem 1.1.

Proof of Theorem 1.1. Noting $\lambda>\Lambda$ by the condition (6) in the statement of Theorem 1.1, we choose $\varepsilon_{0} \in\left(0,3^{-1}(\lambda-\Lambda)\right)$. Since $\Lambda$ is continuous in $M \in$ $[0,1)$, by Lemma 2.1 there exists $M^{\prime} \in(M, 1)$ such that

$$
\begin{aligned}
& \inf _{0<\beta<2-2 M^{\prime}}\left(\sup \left\{\frac{1}{2}\left\{\frac{K^{2}+2 L}{t}+\frac{K^{2}}{2-t}\right\} \mid \beta \leq t \leq \beta+2 M^{\prime}\right\}\right) \\
& \quad<\Lambda+\varepsilon_{0} .
\end{aligned}
$$

Thus there exists some $\beta_{0} \in\left(0,2-2 M^{\prime}\right)$ such that for any $t \in\left[\beta_{0}, \beta_{0}+2 M^{\prime}\right]$ we have

$$
\frac{1}{2}\left\{\frac{K^{2}+2 L}{t}+\frac{K^{2}}{2-t}\right\}<\Lambda+\varepsilon_{0}
$$

Let

$$
\mu_{1}=\liminf _{r \rightarrow \infty} Q(r), \mu_{2}=\limsup _{r \rightarrow \infty} Q(r),
$$

and we have $M=\mu_{2}-\mu_{1}$. Let

$$
\alpha_{0}=\beta_{0}+M^{\prime}-\mu_{1}-\mu_{2}
$$

and we have

$$
\begin{aligned}
& \alpha_{0}+2 \liminf _{r \rightarrow \infty} Q(r)=\alpha_{0}+2 \mu_{1}=\beta_{0}+M^{\prime}-M>\beta_{0}, \\
& \alpha_{0}+2 \underset{r \rightarrow \infty}{\lim \sup } Q(r)=\alpha_{0}+2 \mu_{2}=\beta_{0}+M^{\prime}+M<\beta_{0}+2 M^{\prime} .
\end{aligned}
$$

Therefore there exists $R_{1}>R_{0}$ such that for any $r \geq R_{1}$ we have

$$
0<\beta_{0}<\alpha_{0}+2 Q(r)<\beta_{0}+2 M^{\prime}<2 .
$$

By (2.1), for any $r \geq R_{1}$ we have 


$$
\begin{aligned}
\Lambda+\varepsilon_{0} & >\frac{1}{2}\left\{\frac{K^{2}+2 L}{\alpha_{0}+2 Q(r)}+\frac{K^{2}}{2-\alpha_{0}-2 Q(r)}\right\} \\
& =\frac{L}{\alpha_{0}+2 Q(r)}+\frac{K^{2}}{\left\{\alpha_{0}+2 Q(r)\right\}\left\{2-\alpha_{0}-2 Q(r)\right\}} .
\end{aligned}
$$

By the conditions (2), (3), (4) and (2.2), there exists $R_{2} \geq R_{1}$ such that for any $r \geq R_{2}$ we have

$$
\begin{aligned}
& \Lambda+2 \varepsilon_{0} \\
& \quad>\frac{r \partial_{r} V_{1}(x)}{\alpha_{0}+2 Q(r)}+V_{1}(x)+\frac{\left|r V_{2}(x)\right|^{2}+|B(x) x|^{2}}{\left\{\alpha_{0}+2 Q(r)\right\}\left\{2-\alpha_{0}-2 Q(r)\right\}} .
\end{aligned}
$$

And by (2.2), there exists some constant $C_{3}>0$ such that for any $r \geq R_{2}$ we have

$$
\varepsilon_{0}>\frac{C_{3}\left\{2-\alpha_{0}-2 Q(r)\right\}}{\left\{\alpha_{0}+2 Q(r)\right\}}
$$

Adding above two inequalities and noting $\lambda>\Lambda+3 \varepsilon_{0}$ and (2.2), for any $r \geq R_{2}$ we have

$$
\begin{aligned}
& \frac{1}{2-\alpha_{0}-2 Q(r)}\left[r \partial_{r} V_{1}(x)+\left\{\alpha_{0}+2 Q(r)\right\}\left\{V_{1}(x)-\lambda\right\}\right. \\
& \left.+\frac{1}{2-\alpha_{0}-2 Q(r)}\left\{\left|r V_{2}(x)\right|^{2}+|B(x) x|^{2}\right\}\right] \\
& <-C_{3} .
\end{aligned}
$$

Let

$$
\begin{aligned}
& q_{1}(x)=V_{1}(x)-\lambda, \\
& q_{2}(x)=V_{2}(x)+V_{3}(r) .
\end{aligned}
$$

Then the equation (1.1) coincides with (0.1). Our Assumptions (B)-(D) are satisfied. Now we shall check over all the conditions given in Assumptions (E)(G).

Let for $i=1,2$

$$
\begin{aligned}
& \sigma_{i}(r)=2-\alpha_{0}-2 Q(r), \\
& \eta_{i}(r)=\alpha_{0}+2 Q(r), \\
& \tau(r)=2^{-1} \beta_{0}, \\
& \psi_{i}(r) \equiv 1, \\
& Q_{0}(x)=Q(r), \\
& p(r) \equiv C_{4},
\end{aligned}
$$

where $C_{4}>0$ is a sufficiently large constant. Then we have

$$
\begin{aligned}
& Q_{i}(r)=2^{-1}\left(\alpha_{0}-1\right)+Q(r), \\
& \sigma_{i}(r)+\eta_{i}(r)=2, \\
& \Phi_{1}(r)=r R_{0}^{-1} .
\end{aligned}
$$


Noting (2.2) it can be easily seen that (E) and (G) except (E.7) are satisfied. By (2.2), for any $\mathrm{t} \geq R_{2}$ we have $\tau(t)-\eta_{2}(t) \leq-2^{-1} \beta_{0}$, which leads us to (E.7). By (2.3), (F.2) holds with $a_{i}=1$. By Arai-Uchiyama [2, Remark 1.6] we can see that (F.2) with (F.1) holds.

Now we assume that $\operatorname{supp}[u]$ is not a compact set in $\bar{\Omega}$. Then by Theorem 0.2 we have $u(x) \notin L^{2}(\Omega)$, which contradicts with the assumptions of Theorem 1.1. Thus $\operatorname{supp}[u]$ is a compact set in $\bar{\Omega}$. Since the unique continuation theorem holds to our problem (, see e.g. Garofalo-Lin [4, Theorem 1.1]), we have $u(x) \equiv 0$ on $\Omega$.

\section{§3. Proof of Theorem 1.3}

Proof of Theorem 1.3. If $\zeta_{2}(r)$ satisfies the assumptions, then for $\varepsilon_{1}>0$, which will be determined later, $\zeta_{2}(r)$ replaced with $\zeta_{2}(r)+r^{-1-\epsilon_{1}}$ also satisfies all the conditions given in Theorem 1.3. Thus we can assume, without loss of generality, that for any $r \geq R_{0}$ we have

$$
\zeta_{2}(r) \geq r^{-1-\varepsilon_{1}}
$$

By (iii) and positivity of $\lambda-V_{1}(x)$, there exist constants $C_{5}>0$ and $C_{6}>0$ such that for any $r \geq R_{0}$

$$
\lambda-V_{1}(x) \geq C_{5} r^{2(\alpha-1)} \exp \left(-\int_{R_{0}}^{r} \zeta_{1}(t) d t\right) \geq C_{6} r^{2(\alpha-1)},
$$

where in the last inequality we used (i).

We put

$$
q_{1}(x)=V_{1}(x)-\lambda, q_{2}(x)=V_{2}(x)+V_{3}(r) .
$$

Then the equation (1.2) coincides with (0.1). Our Assumptions (B)-(D) are satisfied.

Let $\varepsilon_{2}>0$ and $\varepsilon_{3}>0$ be constants determined later and we put

$$
\begin{aligned}
& \sigma_{i}(r)=\left(1+2 \varepsilon_{2}\right) r\left\{\zeta_{1}(r)+\zeta_{2}(r)\right\}-2 Q(r)(i=1,2), \\
& \eta_{1}(r)=2(1-\alpha)+\left(1+2 \varepsilon_{2}\right) r\left\{\zeta_{1}(r)+\zeta_{2}(r)\right\}+2 Q(r), \\
& \eta_{2}(r)=2(1-\alpha)+\varepsilon_{3}+\left(1+2 \varepsilon_{2}\right) r\left\{\zeta_{1}(r)+\zeta_{2}(r)\right\}+2 Q(r), \\
& \psi_{i}(r)=r^{1-\alpha}(i=1,2) .
\end{aligned}
$$

By (ii), (vi), (vii) and (3.1) there exist some $\varepsilon_{2}>0, \varepsilon_{3}>0, C_{7}>0$ and $R_{3} \geq R_{0}$ such that for any $r \geq R_{3}$ we have

$$
\begin{aligned}
& 2-\eta_{i}(r) \geq C_{7}(i=1,2), \\
& |Q(r)| \leq 2^{-1} \varepsilon_{2} r\left\{\zeta_{1}(r)+\zeta_{2}(r)\right\}, \\
& r^{-\varepsilon_{1}} \leq\left(1+\varepsilon_{2}\right) r\left\{\zeta_{1}(r)+\zeta_{2}(r)\right\} \leq \sigma_{i}(r) \leq\left(1+3 \varepsilon_{2}\right) r\left\{\zeta_{1}(r)+\zeta_{2}(r)\right\} .
\end{aligned}
$$


So the Assumptions (E.1)-(E.3) are satisfied. Let $0<\varepsilon_{1}<\min \left\{\alpha, \varepsilon_{3}\right\}, 0<\tau_{0}<\varepsilon_{3}$ $-\varepsilon_{1}$ and $\tau(t) \equiv \tau_{0}$. By (3.5), we have (E.4). (E.5) is obvious since $\sigma_{1}=\sigma_{2}$ and $\eta_{1}-\eta_{2}$ is a constant. (E.6) is also obvious by (ii) and (3.5). By (3.4) and non-negativities of $\zeta_{1}(r)$ and $\zeta_{2}(r)$ we have $\tau(t)-\eta_{2}(t) \leq \tau_{0}-2(1-\alpha)-\varepsilon_{3}$ for any $t \geq R_{3}$. Then by (3.5)

$$
\psi_{2}^{2} \sigma_{2}^{-1} \exp \left(\int_{R_{0}}^{r} \frac{\tau(t)-\eta_{2}(t)}{t} d t\right) \leq \text { Const } r^{\tau_{0}+\varepsilon_{1}-\varepsilon_{3}}
$$

which leads us to (E.7).

Now we shall show (F.2). By (iii), (3.4) and (3.5), we have for any $r \geq R_{3}$

$$
\begin{aligned}
\sigma_{i}^{-1}\left(r \partial_{r} q_{1}+\eta_{i} q_{1}\right) \\
=-r \sigma_{i}^{-1}\left[\left\{-\partial_{r} V_{1}+\left(\frac{2(1-\alpha)}{r}+\zeta_{1}\right)\left(\lambda-V_{1}\right)\right\}\right. \\
\left.\quad+\left\{\zeta_{2}+2 \varepsilon_{2}\left(\zeta_{1}+\zeta_{2}\right)+\varepsilon_{3} \delta_{i 2} r^{-1}+2 r^{-1} Q\right\}\left(\lambda-V_{1}\right)\right] \\
\leq-r \sigma_{i}^{-1}\left\{\zeta_{2}+\varepsilon_{2}\left(\zeta_{1}+\zeta_{2}\right)\right\}\left(\lambda-V_{1}\right) \\
\leq-\left\{r \sigma_{i}^{-1} \zeta_{2}+\varepsilon_{2}\left(1+3 \varepsilon_{2}\right)^{-1}\right\}\left(\lambda-V_{1}\right),
\end{aligned}
$$

where $\delta_{i j}$ is Kronecker's delta. Let $a_{i}=1+\varepsilon_{2}(>1)$. By (3.5) and non-negativity of $\zeta_{1}(r)$ we have $a_{i} r \zeta_{2} \leq \sigma_{i}$ for any $r \geq R_{3}$. Note that $Q_{i}=4^{-1}\left(\eta_{i}-\sigma_{i}\right)=Q(r)$ $+2^{-1}(1-\alpha)+4^{-1} \varepsilon_{3} \delta_{i 2}$. Then by (iv) and (v) we have for any $r \geq R_{3}$

$$
a_{i} \sigma_{i}^{-2}\left|r q_{2}-Q_{i}^{\prime}\right|^{2}=a_{i} r^{2} \sigma_{i}^{-2}\left|V_{2}\right|^{2} \leq a_{i} r^{2} \sigma_{i}^{-2} \zeta_{2}^{2}\left(\lambda-V_{1}\right) \leq r \sigma_{i}^{-1} \zeta_{2}\left(\lambda-V_{1}\right) .
$$

By (vii), (3.3) and $\sigma_{i} \geq r\left(\zeta_{1}+\zeta_{2}\right)$ we have for any $r \geq R_{3}$

$$
\sigma_{i}^{-1}\left(2-\eta_{i}\right)^{-1}|B x|^{2} \leq C_{7}^{-1} \mu(r)\left(\lambda-V_{1}\right) .
$$

Thus, noting (3.2), for any $r \geq R_{3}$ we have

$$
\begin{aligned}
& \psi_{i}^{2} \sigma_{i}^{-1}\left[r \partial_{r} q_{1}+\eta_{i} q_{1}+a_{i} \sigma_{i}^{-1}\left|r q_{2}-Q_{i}^{\prime}\right|^{2}+\left(2-\eta_{i}\right)^{-1}|B x|^{2}\right] \\
& \quad \leq-r^{2-2 \alpha}\left\{\varepsilon_{2}\left(1+3 \varepsilon_{2}\right)^{-1}-C_{7}^{-1} \mu(r)\right\}\left(\lambda-V_{1}\right) \\
& \quad \leq-C_{6}\left\{\varepsilon_{2}\left(1+3 \varepsilon_{2}\right)^{-1}-C_{7}^{-1} \mu(r)\right\},
\end{aligned}
$$

which and (viii) lead us to (F.2).

Since unique continuation theorem can be applied to our problem (1.2) and $u \neq \equiv$, we can admit that $\operatorname{supp}[u]$ is not a compact set. Thus we can apply Theorem 0.1 and, using positivity of $\lambda-V_{1}(x)$, we obtain

$$
\liminf _{R \rightarrow \infty} \int_{|x|=R} \Phi_{1}(r)\left[|D u|^{2}+\left\{r^{-2}+\left(\lambda-V_{1}(x)\right)\right\}|u|^{2}\right] d S>0,
$$

where $\Phi_{1}(r)=\exp \left(\int_{R_{0}}^{r} \frac{\sigma_{1}(t)+\eta_{1}(t)}{2 t} d t\right) \leq C_{8} r^{1-\alpha}$ by (i) with some positive constant $C_{8}$. Now by (ii) and (3.2) there exist some constants $R_{4} \geq R_{3}$ and $C_{9}>$ 0 such that for any $r \geq R_{4}$ we have 


$$
\begin{aligned}
& r^{2}\left(\lambda-V_{1}(x)\right) \geq \text { Const }>0, \\
& \int_{|x|=r}\left[|D u|^{2}+\left(\lambda-V_{1}(x)\right)|u|^{2}\right] d S \geq C_{9} r^{\alpha-1}, \\
& \zeta_{2}(r)<2 \alpha r^{-1} .
\end{aligned}
$$

By (iv), (v), (3.6) and (3.8), we have

$$
\left|q_{2}-r^{-1} Q^{\prime}\right|=\left|V_{2}\right| \leq 2^{-1}\left\{\zeta_{2}^{2}+\left(\lambda-V_{1}\right)\right\} \leq \operatorname{Const}\left(\lambda-V_{1}\right) .
$$

Thus $\tilde{p}(x)=\operatorname{Const}\left(\lambda-V_{1}(x)\right)$ and $Q_{0}(x)=Q(r)$ satisfy the assumptions of Arai-Uchiyama [2, Lemma 6.2] and there exist some $R_{5} \geq R_{4}$ and $C_{10}>0$ such that for any $\varphi(r) \in C_{0}^{1}\left(R_{5}, \infty\right)$ we have

$$
\begin{aligned}
& \int_{\Omega} \varphi(r)^{2}\left[|D u|^{2}+\left(\lambda-V_{1}(x)\right)|u|^{2}\right] d x \\
& \quad \leq C_{10} \int_{\Omega}\left\{\left(\lambda-V_{1}(x)\right) \varphi(r)^{2}+\varphi^{\prime}(r)^{2}\right\}|u|^{2} d x .
\end{aligned}
$$

Let $\varphi_{1}(t) \in C_{0}^{\infty}(\boldsymbol{R})$ be a function satisfying $0 \leq \varphi_{1}(t) \leq 1$ in $(-\infty, \infty), \varphi_{1}(t)$ $=0$ for $t \leq 1 / 4$ and $t \geq 1$, and $\varphi_{1}(t)=1$ for $1 / 2 \leq t \leq 3 / 4$. Let $R>4 R_{5}$ and put $\varphi_{R}(r)=\varphi_{1}(r / R)$. Then for any $R>4 R_{5}, \varphi_{R}(r) \in C_{0}^{1}\left(R_{5}, \infty\right)$ and (3.7) holds for any $r>R / 2$. Integrate both sides of (3.7) in $R / 2<r<(3 R) / 4$. Then there exists some $C_{11}>0$ such that for any $R>4 R_{5}$ we have

$$
\begin{aligned}
C_{11} R^{\alpha} & \leq \int_{\frac{R}{2}<|x|<\frac{3}{4} R}\left[|D u|^{2}+\left(\lambda-V_{1}(x)\right)|u|^{2}\right] d x \\
& \leq \int_{\Omega} \varphi_{R}(|x|)^{2}\left[|D u|^{2}+\left(\lambda-V_{1}(x)\right)|u|^{2}\right] d x \\
& \left.\leq C_{10} \int_{\Omega}\left\{\left(\lambda-V_{1}(x)\right) \varphi_{R}(r)^{2}+\varphi_{R}^{\prime}(r)^{2}\right\}|u|^{2}\right] d x \\
& \leq \text { Const } \int_{R_{0}<|x|<R}\left(\lambda-V_{1}(x)\right)|u|^{2} d x,
\end{aligned}
$$

where in the last inequality, noting (3.6), we have used $\varphi_{R}^{\prime}(r)^{2} \leq$ Const $r^{-2} \leq$ Const $\left(\lambda-V_{1}(x)\right)$. Thus we have (1.3).

\section{References}

[1] Agmon, S., Lower bounds for solutions of Schrödinger equations, J. Analyse Math., 23(1970), 1-25.

[2] Arai, M. and Uchiyama, J., Growth order of eigenfunctions of Schrödinger operators with potentials admitting some integral conditions I - General theory-, Publ. RIMS, Kyoto Univ., 32 (1996), 581-616.

[3] Eastham, M.S.P. and Kalf, H., Schrödinger-type operators with continuous spectra. Research Notes in Mathematics 65, Pitman, Boston, London, Melbourne, 1982.

[4] Garofalo, N. and Lin, F.H., Unique continuation for elliptic operators: A geometric- 
variational approach, Comm. Pure Appl. Math., 40 (1987), 347-366.

[5] Kalf, H. and Kumar, V.K., On the absence of positive eigenvalues of Schrödinger operators with long range potentials, Trans. Amer. Math. Soc., 275 (1983), 215-229.

[6] Kato, T., Growth properties of solutions of the reduced wave equation with variable coefficients, Comm. Pure Appl. Math., 12 (1959), 403-425.

[7] Khosrovshahi, G.B., Levine, H.A. and Payne, L.E., On the positive spectrum of Schrödinger operators with long range potentials, Trans. Amer. Math. Soc., 253 (1979), 211-228.

[8] von Neumann, J. and Wigner, E.P., Über merkwürdige diskrete eigenwerte, Phys. Z., 30 (1929), 465-467.

[9] Uchiyama, J. and Yamada, O., Sharp estimates of lower bounds of polynomial decay order of eigenfunctions, Publ. RIMS, Kyoto Univ., 26 (1990), 419-449. 
